Bull. Soc. math. France

132 (2), 2004, p. 305-326

\title{
EXPONENTIALS FORM A BASIS OF DISCRETE HOLOMORPHIC FUNCTIONS ON A COMPACT
}

\author{
By Christian Mercat
}

\begin{abstract}
We show that discrete exponentials form a basis of discrete holomorphic functions on a finite critical map. On a combinatorially convex set, the discrete polynomials form a basis as well.

RÉSUMÉ (Les exponentielles forment une base des fonctions holomorphes discrètes sur un compact)

Nous montrons que les exponentielles forment une base des fonctions holomorphes discrètes sur une carte critique compacte. Sur un convexe, les polynômes discrets forment également une base.
\end{abstract}

\section{Introduction}

The notion of discrete Riemann surfaces has been defined in [13]. A good basis for the associated space of holomorphic functions was still missing. This article discuss an interesting one in the simply connected critical case.

Texte reçu le 15 janvier 2003, accepté le 16 juin 2003

Christian Mercat, Sfb 288, MA 8-5, Technische Universität, Strasse des 17. Juni 136, D10623 Berlin (Germany) • Département de mathématiques, Université de Montpellier II, C.C. 51, F-34095 Montpellier cedex 5 (France) • E-mail : mercat@math.univ-montp2.fr 2000 Mathematics Subject Classification. - 30G25, 52C26, 31C20, 39A12.

Key words and phrases. - Discrete holomorphic functions, discrete analytic functions, monodriffic functions, exponentials.

This research is supported by the Deutsche Forschungsgemeinschaft in the framework of Sonderforschungsbereich 288, "Differential Geometry and Quantum Physics". I thank Richard Kenyon for his comments on the draft of this article. 


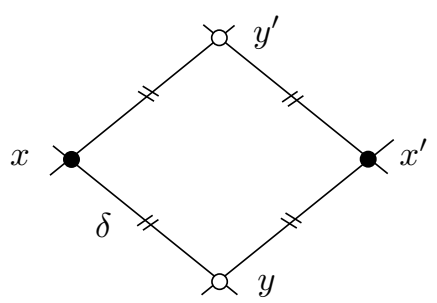

FiguRE 1. The discrete Cauchy-Riemann equation takes place on each rhombus.

We are interested in a cellular decomposition $\diamond$ of the complex plane or a simply connected portion $U$ of it, by rhombi (equilateral quadrilaterals, or lozenges). In other words, our cellular complex is made of quadrilaterals (a quad-graph) and we have a map from the set of vertices $\nabla_{0}$ to the complex plane $Z: \diamond_{0} \rightarrow \mathbb{C}$ such that for each oriented face $\left(x, y, x^{\prime}, y^{\prime}\right) \in \diamond_{2}$, its image is a positively oriented rhombus $\left(Z(x), Z(y), Z\left(x^{\prime}\right), Z\left(y^{\prime}\right)\right)$ of side length $\delta>0$. It defines a straightforward Cauchy-Riemann equation for a function $f \in C^{0}(\diamond)$ of the vertices, and similarly for 1-forms:

$$
\frac{f\left(y^{\prime}\right)-f(y)}{Z\left(y^{\prime}\right)-Z(y)}=\frac{f\left(x^{\prime}\right)-f(x)}{Z\left(x^{\prime}\right)-Z(x)} .
$$

We call such a data a critical map of $U$. The relevance of this kind of maps in the context of discrete holomorphy was first pointed out and put to use by Duffin [8]. The rhombi can be split in four, yielding a finer critical map $\diamond^{\prime}, Z^{\prime}$ with $\delta^{\prime}=\frac{1}{2} \delta$. In [13], [12] we proved that a converging sequence of discrete holomorphic functions on a refining sequence of critical maps converges to a continuous holomorphic function and any holomorphic function on $U$ can be approximated by a converging sequence of discrete holomorphic functions. The proof was based on discrete polynomials and series. In the present article we are going to show that the vector space spanned by discrete polynomials is the same as the one spanned by discrete exponentials and the main result is the following

THEOREM 1. - On a combinatorially convex finite critical map, the discrete exponentials form a basis of discrete holomorphic functions.

On a non combinatorially convex map we define some special exponentials which supplement this basis.

The article is organized as follows. After recalling some basic features of discrete Riemann surfaces at criticality in Section 2, we define discrete exponentials in Section 3 and show some of their basic properties, related to polynomials and series. We give in particular a formula for the expression of a generic exponential in a basis of exponentials. In Section 4, we introduce the notion of convexity, related to a geometrical construction called train-tracks, and we

TOME $132-2004-\mathrm{N}^{\mathrm{O}} 2$ 
prove the main result. Finally we study the general case in Section 5 where we define special exponentials and show they form a basis. The appendix lists some other interesting properties of the discrete exponentials which are not needed in the proof.

We note that it is possible to use the wonderful machinery defined in [10] to prove that discrete exponentials form a basis of discrete holomorphic functions on a critical compact. Indeed, Richard Kenyon gives an expression of the discrete Green's function (the discrete logarithm) as an integral over a loop in the space of discrete exponentials:

$$
G(O, x)=-\frac{1}{8 \pi^{2} i} \oint_{C} \exp (: \lambda: x) \frac{\log \frac{1}{2} \delta \lambda}{\lambda} \mathrm{d} \lambda
$$

where the integration contour $C$ contains all the points in $P_{\diamond}$ (the possible poles of $\exp (: \lambda: x))$ but avoids the half line through $-x$. It is real (negative) on half of the vertices and imaginary on the others. Because of the logarithm, this imaginary part is multivalued, it has a (discrete) logarithmic singularity at the origin: the Laplacian of $G(O, \bullet)$ is 1 there, and null elsewhere. On a compact, considering points on the boundary as origins, these functions can be single valued and can be formed into a basis of discrete holomorphic functions. They clearly belong in the space of discrete exponentials.

The approach we will present here is much more pedestrian and simplistic.

In a forthcoming paper in collaboration with A. Bobenko, B. Springborn and Y.Suris, the theory will be generalized to a much broader setup, based on a quadratic notion of discrete holomorphicity: cross-ratio preserving maps. Given a base function $F$ defined on a quad-graph, a function $G$ is cross-ratio holomorphic [3], [2], [4] if, for every quadrilateral $\left(x, y, x^{\prime}, y^{\prime}\right) \in \diamond_{2}$,

$$
\frac{G(x)-G(y)}{G(y)-G\left(x^{\prime}\right)} \cdot \frac{G\left(x^{\prime}\right)-G\left(y^{\prime}\right)}{G\left(y^{\prime}\right)-G(x)}=\frac{F(x)-F(y)}{F(y)-F\left(x^{\prime}\right)} \cdot \frac{F\left(x^{\prime}\right)-F\left(y^{\prime}\right)}{F\left(y^{\prime}\right)-F(x)} .
$$

The circle packings of a given combinatorics and intersection angles form a very interesting example of such cross-ratio preserving maps.

Infinitesimal deformations of a function $F$ with given cross-ratios, that preserve these cross-ratios, are parametrized by the vector space $\Omega(F)$ of discrete linear holomorphic functions $g$ defined by the fact that their ratios along the diagonals of the quadrilaterals are the same as the base function: $g$ is linear holomorphic at $F$ if

$$
\frac{g(x)-g\left(x^{\prime}\right)}{g(y)-g\left(y^{\prime}\right)}=\frac{F(x)-F\left(x^{\prime}\right)}{F(y)-F\left(y^{\prime}\right)} .
$$

An analytic vector field of linear holomorphic functions can be integrated into cross-ratio preserving maps. For example, the vector field given by the Green function is integrated into the 1-parameter line $Z^{\gamma}[1]$. 
An important classical ingredient in the theory of cross-ratio preserving maps is the Bäcklund transformation [4], [5], [9]. It is a two complex parameters $(u, \lambda)$ family of (cross-ratio) holomorphic functions $B_{\lambda}^{u}(F)$, that is to say with the same cross-ratios as $F$. The parameter $u$ is a starting value at a given origin, $B_{\lambda}^{u}(F)(O)=u$. This transformation verifies

$$
B_{-\lambda}^{F(O)}\left(B_{\lambda}^{u}(F)\right)=F
$$

for any $(u, \lambda)$, and the identity transformation corresponds to $(u, \lambda)=$ $(F(O), 0), B_{0}^{F(O)}(F)=F$. It is an anlytic transformation in all the parameters therefore its derivative is a linear map between the tangent spaces,

$$
\mathrm{d} B_{\lambda}^{u}(F): \Omega(F) \longrightarrow \Omega\left(B_{\lambda}^{u}(F)\right) .
$$

It is not injective and I define the discrete exponential at $F$ as being the direction of this 1-dimensional kernel. It can be characterized as a derivative with respect to the initial value at the origin:

$$
\exp _{u}(: \lambda: F):=\frac{\partial}{\partial v} B_{-\lambda}^{v}\left(B_{\lambda}^{u}(F)\right)_{\mid v=F(O)} \in \operatorname{ker}\left(\mathrm{d} B_{\lambda}^{u}(F)\right)
$$

because $B_{\lambda}^{u}\left(B_{-\lambda}^{v}(G)\right)=G$ for all $\lambda, G$ and $v$. We use them to derive Kenyon's formula in a more general setup and cover the subject of an infinite critical map with finitely many slopes, extending the results of the present article.

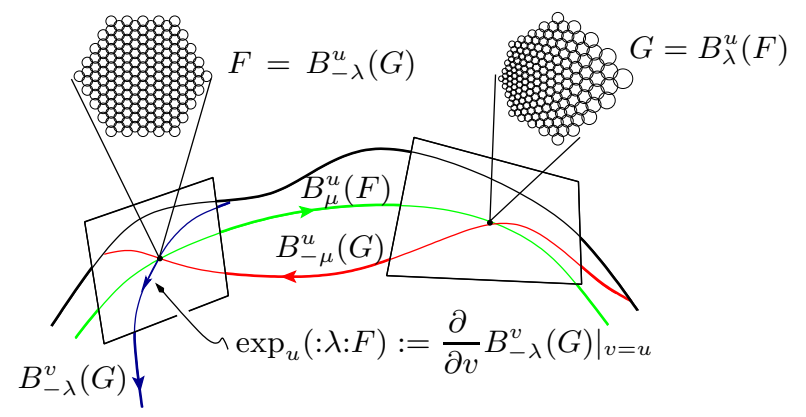

Figure 2. The discrete exponential $\exp _{u}(: \lambda: F)$ is the kernel of the linear transformation $\mathrm{d} B_{\lambda}^{u}(F)$ (here $u=F(O)$ ).

\section{Integration and Derivation at criticality}

2.1. Integration. - Given an isometric local map $Z: U \cap \diamond \rightarrow \mathbb{C}$, where the image of the quadrilaterals are lozenges in $\mathbb{C}$, any holomorphic function $f \in \Omega(\diamond)$ gives rise to an holomorphic 1-form $f \mathrm{~d} Z$ defined by the formula,

$$
\int_{(x, y)} f \mathrm{~d} Z:=\frac{f(x)+f(y)}{2}(Z(y)-Z(x)),
$$

TOME $132-2004-\mathrm{N}^{\mathrm{O}} 2$ 
where $(x, y) \in \diamond_{1}$ is an edge of a lozenge. It fulfills the Cauchy-Riemann equation for forms which is, in the same conditions as Eq. (1.1):

$$
\begin{aligned}
& \frac{1}{Z\left(y^{\prime}\right)-Z(y)}\left(\int_{(y, x)}+\int_{\left(x, y^{\prime}\right)}+\int_{\left(y, x^{\prime}\right)}+\int_{\left(x^{\prime}, y^{\prime}\right)}\right) f \mathrm{~d} Z \\
& =\frac{1}{Z\left(x^{\prime}\right)-Z(x)}\left(\int_{(x, y)}+\int_{\left(y, x^{\prime}\right)}+\int_{\left(x, y^{\prime}\right)}+\int_{\left(y^{\prime}, x^{\prime}\right)}\right) f \mathrm{~d} Z .
\end{aligned}
$$

Once an origin $O$ is chosen, it provides a way to integrate a function $\operatorname{Int}(f)(z):=\int_{O}^{z} f \mathrm{~d} Z$. We proved in [12] that the integrals of converging discrete holomorphic functions $\left(f^{k}\right)_{k}$ on a refining sequence $\left(\diamond^{k}\right)_{k}$ of critical maps of a compact converge to the integral of the limit. If the original limit was of order $f(z)=f^{k}(z)+O\left(\delta_{k}^{2}\right)$, it stays this way for the integrals, $\int_{O}^{z} f(u) \mathrm{d} u=\int_{O}^{z} f^{k} \mathrm{~d} Z+O\left(\delta_{k}^{2}\right)$, where the left hand side is the usual continuous integral and the right hand side the discrete ones.

Following Duffin [7], [8], we define by inductive integration the discrete ana$\operatorname{logues}$ of the integer power monomials $z^{k}$, that we denote $Z^{: k}$ :

$$
\begin{aligned}
& Z^{: 0:}:=1, \\
& Z^{: k}:=k \int_{O} Z^{: k-1:} \mathrm{d} Z .
\end{aligned}
$$

The discrete polynomials of degree less than three agree point-wise with their continuous counterpart, $Z^{: 2:}(x)=Z(x)^{2}$ so that by repeated integration, the discrete polynomials in a refining sequence of a compact converge to the continuous ones and the limit is of order $O\left(\delta^{2}\right)$. We will see (Eq. (3.6)) that a closed formula can be obtained for these monomials.

2.2. Derivation. - The combinatorial surface being simply connected and the graph $\diamond$ having only quadrilateral faces, it is bi-colorable. Let $\Gamma$ and $\Gamma^{*}$ the two sets of vertices and $\varepsilon$ be the biconstant $\varepsilon(\Gamma)=+1, \varepsilon\left(\Gamma^{*}\right)=-1$. For a holomorphic function $f$, the equality $f \mathrm{~d} Z \equiv 0$ is equivalent to $f=\lambda \varepsilon$ for some $\lambda \in \mathbb{C}$.

Following Duffin [7], [8], we introduce the

Definition 2.1. - For a holomorphic function $f$, define on a flat simply connected map $U$ the holomorphic functions $f^{\dagger}$, the $d u a l$ of $f$, and $f^{\prime}$, the derivative of $f$, by the formulae

$$
f^{\dagger}(z):=\varepsilon(z) \bar{f}(z),
$$

where $\bar{f}$ denotes the complex conjugate, $\varepsilon= \pm 1$ is the biconstant, and

$$
f^{\prime}(z):=\frac{4}{\delta^{2}}\left(\int_{O}^{z} f^{\dagger} \mathrm{d} Z\right)^{\dagger}+\lambda \varepsilon
$$

defined up to $\varepsilon$. 
We proved in [13] the following

Proposition 2.2. - The derivative $f^{\prime}$ fulfills

$$
\mathrm{d} f=f^{\prime} \mathrm{d} Z \text {. }
$$

\section{Exponential}

\subsection{Definition}

Definition 3.1. - The discrete exponential $\exp (: \lambda: Z)$ is the solution of

$$
\exp (: \lambda: O)=1, \quad \mathrm{~d} \exp (: \lambda: Z)=\lambda \exp (: \lambda: Z) \mathrm{d} Z .
$$

We define its derivatives with respect to the continuous parameter $\lambda$ :

$$
Z^{: k:} \exp (: \lambda: Z):=\frac{\partial^{k}}{\partial \lambda^{k}} \exp (: \lambda: Z)
$$

The discrete exponential was first defined in [11] and put to a very interesting use in [10]. For $|\lambda| \neq 2 / \delta$, an immediate check shows that it is a rational fraction in $\lambda$ at every point: For the vertex $x=\sum \delta \mathrm{e}^{i \theta_{k}}$,

$$
\exp (: \lambda: x)=\prod_{k} \frac{1+\frac{1}{2} \lambda \delta \mathrm{e}^{i \theta_{k}}}{1-\frac{1}{2} \lambda \delta \mathrm{e}^{i \theta_{k}}}
$$

where $\left(\theta_{k}\right)$ are the angles defining $\left(\delta \mathrm{e}^{i \theta_{k}}\right)$, the set of $(Z$-images of $) \diamond$-edges between $x$ and the origin. Because the map is critical, Eq. (3.3) only depends on the end points $(O, x)$. It is a generalization of a well known formula, in a slightly better version,

$$
\exp (\lambda x)=\left(1+\frac{\lambda x}{n}\right)^{n}+O\left(\frac{\lambda^{2} x^{2}}{n}\right)=\left(\frac{1+\lambda x / 2 n}{1-\lambda x / 2 n}\right)^{n}+O\left(\frac{\lambda^{3} x^{3}}{n^{2}}\right)
$$

to the case when the path from the origin to the point $x=\sum_{1}^{n} x / n=\sum \delta \mathrm{e}^{i \theta_{k}}$ is not restricted to straight equal segments but to a general path of $O(|x| / \delta)$ segments of any directions.

The integration with respect to $\lambda$ gives an interesting analogue of $Z^{:-k:} \exp (: \lambda: Z)$. It is defined up to a globally defined discrete holomorphic map. One way to fix it is to integrate from a given $\lambda_{0}$ of modulus $2 / \delta$, which is not a pole of the rational fraction, along a path that doesn't cross the circle of radius $2 / \delta$ again.

Proposition 3.2. - For point-wise multiplication, at every point $x \in \diamond_{0}$,

$$
\exp (: \lambda: x) \cdot \exp (:-\lambda: x)=1 \text {. }
$$

The specialization at $\lambda=0$ gives back the monomials:

$$
Z^{: k:}=Z^{: k:} \exp (: \lambda: Z)_{\mid \lambda=0}
$$

TOME $132-2004-\mathrm{N}^{\mathrm{O}} 2$ 
The anti-linear duality $\dagger$ maps exponentials to exponentials:

$$
\exp (: \lambda:)^{\dagger}=\exp \left(: \frac{4}{\delta^{2} \bar{\lambda}}:\right)
$$

In particular, $\exp (: \infty:)=1^{\dagger}=\varepsilon$ is the biconstant.

Proof. - The first assertion is immediate. The derivation of (3.1) with respect to $\lambda$ yields

$$
\mathrm{d} \frac{\partial^{k}}{\partial \lambda^{k}} \exp (: \lambda: Z)=\left(\lambda \frac{\partial^{k}}{\partial \lambda^{k}} \exp (: \lambda: Z)+k \frac{\partial^{k-1}}{\partial \lambda^{k-1}} \exp (: \lambda: Z)\right) \mathrm{d} Z
$$

which implies (3.6). Derivation of $\exp (: \lambda:)^{\dagger}$ gives,

$$
\begin{aligned}
\left(\exp (: \lambda:)^{\dagger}\right)^{\prime} & =\frac{4}{\delta^{2}}\left(\int_{O}^{z} \exp (: \lambda:) \mathrm{d} Z\right)^{\dagger}+\mu \varepsilon \\
& =\frac{4}{\delta^{2}}\left(\frac{\exp (: \lambda:)-1}{\lambda}\right)^{\dagger}+\mu \varepsilon=\frac{4}{\delta^{2} \bar{\lambda}} \exp (: \lambda:)^{\dagger}+\nu \varepsilon
\end{aligned}
$$

with $\mu, \nu$ some constants, so that the initial condition $\exp (: \lambda: O)^{\dagger}=1$ at the origin and the difference equation $\mathrm{d} \exp (: \lambda:)^{\dagger}=4 /\left(\delta^{2} \bar{\lambda}\right) \exp (: \lambda:)^{\dagger} \mathrm{d} Z$ yields the result.

Note that it is natural to define $\exp \left(: \lambda:\left(x-x_{0}\right)\right):=\exp (: \lambda: x) / \exp \left(: \lambda: x_{0}\right)$ as a function of $x$ with $x_{0}$ a fixed vertex. It is simply a change of origin. But apart on a lattice where addition of vertices or multiplication by an integer can be given a meaning as maps of the lattice, there is no easy way to generalize this construction to other discrete holomorphic functions such as $\exp (: \lambda:(x+n y))$ with $x, y \in \nabla_{0}$ and $n \in \mathbb{Z}$.

3.2. Series. - The series $\sum_{k=0}^{\infty} \lambda^{k} Z^{: k} / k$ !, wherever it is absolutely convergent, coincide with the rational fraction (3.3): Its value at the origin is 1 and it fulfills the defining difference equation (3.1). Using Eq. (3.6), a Taylor expansion of $\exp (: \lambda: x)$ at $\lambda=0$ gives back the same result. We are now interested in the rate of growth of the monomials.

Because $Z^{: k:} / k$ ! are the iterations of the integration operator Int on the constant function 1, their norm can not grow faster than the powers of its largest eigenvalue $\lambda_{\max }$, which implies absolute convergence for $|\lambda|<1 /\left|\lambda_{\max }\right|$. We have some information on these eigenvalues, summarized in the appendix, through the minimal polynomial of $Z$.

Direct analysis gives an estimate of $Z^{: k}$ :

Proposition 3.3. - For $x \in \diamond$, at a combinatorial distance $d(x, O)$ of the origin, and any $k \in \mathbb{N}$,

$$
\left|\frac{Z^{: k:}(x)}{k !}\right| \leq\left(\frac{\alpha+1}{\alpha-1}\right)^{d(x, O)}\left(\alpha \frac{\delta}{2}\right)^{k},
$$

BULLETIN DE LA SOCIÉtÉ MATHÉMATIQUE DE FRANCE 
for any $\alpha>1$ arbitrarily close to 1 .

Corollary 3.4. - The series $\sum_{k=0}^{\infty} \lambda^{k} Z^{: k} / k !$ is absolutely convergent for $|\lambda|<2 / \delta$.

Proof of Proposition 3.3. - It is proved by double induction, on the degree $k$ and on the combinatorial distance to the origin. For $k=0$, it is valid for any $x$ since $(\alpha+1) /(\alpha-1)=1+2 /(\alpha-1)>1$, with equality only at the origin.

Consider $x \in \diamond$ a neighbor of the origin, $Z(x)=\delta \mathrm{e}^{i \theta}$, then an immediate induction gives for $k \geq 1$,

$$
\frac{Z^{: k:}(x)}{k !}=2\left(\frac{\delta \mathrm{e}^{i \theta}}{2}\right)^{k}
$$

which fulfills the condition Eq. (3.10) for any $k \geq 1$ because $(\alpha+1) /(\alpha-1) \alpha^{k}>2$. This was done merely for illustration purposes since it is sufficient to check that the condition holds at the origin, which it obviously does.

Suppose the condition is satisfied for a vertex $x$ up to degree $k$, and for its neighbor $y$, one edge further from the origin, up to degree $k-1$. Then,

$$
\frac{Z^{: k:}(y)}{k !}=\frac{Z^{: k:}(x)}{k !}+\frac{Z^{: k-1:}(x)+Z^{: k-1:}(y)}{(k-1) !} \cdot \frac{Z(y)-Z(x)}{2}
$$

in absolute value fulfills

$$
\begin{aligned}
\left|\frac{Z^{: k:}(y)}{k !}\right| & \leq\left(\frac{\alpha+1}{\alpha-1}\right)^{d(x, O)}\left(\alpha \frac{\delta}{2}\right)^{k-1}\left\{\left(\alpha \frac{\delta}{2}\right)+\left(1+\frac{\alpha+1}{\alpha-1}\right) \frac{\delta}{2}\right\} \\
& =\left(\frac{\alpha+1}{\alpha-1}\right)^{d(x, O)}\left(\alpha \frac{\delta}{2}\right)^{k}\left(1+\frac{2}{\alpha-1}\right) \\
& =\left(\frac{\alpha+1}{\alpha-1}\right)^{d(y, O)}\left(\alpha \frac{\delta}{2}\right)^{k},
\end{aligned}
$$

thus proving the condition for $y$ at degree $k$. It follows by induction that the condition holds at any point and any degree.

\subsection{Basis}

THEOREM 2. - The vector spaces of discrete exponentials and of discrete polynomials coincide. $A$ basis is given by any set of exponentials $\left\{\exp \left(: \lambda_{\ell}:\right)\right\}_{1 \leq \ell \leq n}$ of the right dimension $n=\operatorname{dim} \operatorname{Vect}\left(Z^{: k:}\right)$, with $\lambda_{k} \neq \lambda_{\ell}$, distinct complex values of norm different from $2 / \delta$. In such a basis, if for a fixed $\lambda_{0}$,

$$
\exp \left(: \lambda_{0}:\right)=\sum_{\ell=1}^{n} \mu_{\ell}\left(\lambda_{0}\right) \exp \left(: \lambda_{\ell}:\right)
$$

then $\exp (: \lambda:)$ is expressed as

$$
\exp (: \lambda:)=\left(\sum_{\ell=1}^{n} \frac{\lambda_{0}-\lambda_{\ell}}{\lambda-\lambda_{\ell}} \mu_{\ell}\left(\lambda_{0}\right)\right)^{-1} \sum_{\ell=1}^{n} \frac{\lambda_{0}-\lambda_{\ell}}{\lambda-\lambda_{\ell}} \mu_{\ell}\left(\lambda_{0}\right) \exp \left(: \lambda_{\ell}:\right) .
$$

TOME $132-2004-\mathrm{N}^{\mathrm{O}} 2$ 
Proof of Theorem 2. - An exponential being equal to a series belongs to the space of polynomials. Equation (3.6) proves the reciprocal. Another way to express it is the direct limit

$$
Z^{: k:}=\lim _{\lambda \rightarrow 0} k ! \lambda^{-k}\left(\exp (: \lambda: Z)-\sum_{\ell=0}^{k-1} \frac{\lambda^{\ell} Z^{: \ell:}}{\ell !}\right) .
$$

Let $\left\{\exp \left(: \lambda_{\ell}:\right)\right\}_{0 \leq \ell \leq k}$ a set of non trivial linear dependent exponentials, $\lambda_{\ell} \neq \lambda_{k}$. It can be reduced by iteration of deletions to a single linear combination dependence

$$
\exp \left(: \lambda_{0}:\right)=\sum_{\ell=1}^{k} \mu_{\ell}\left(\lambda_{0}\right) \exp \left(: \lambda_{\ell}:\right)
$$

with $\left\{\exp \left(: \lambda_{\ell}:\right)\right\}_{1 \leq \ell \leq k}$ a free set of at least two elements. We are going to show that it then provides a basis.

We show Eq. (3.15) first for $\lambda=0$. The integral of $\exp \left(: \lambda_{0}:\right)$ is equal to

$$
\int_{O}^{z} \exp \left(: \lambda_{0}:\right)=\frac{\exp \left(: \lambda_{0}:\right)-1}{\lambda_{0}}=\sum_{\ell=1}^{k} \mu_{\ell}\left(\lambda_{0}\right) \frac{\exp \left(: \lambda_{\ell}:\right)-1}{\lambda_{\ell}}
$$

so that

$$
\sum_{\ell=1}^{k} \frac{\lambda_{0}}{\lambda_{\ell}} \mu_{\ell}\left(\lambda_{0}\right) \exp \left(: \lambda_{\ell}:\right)=\exp \left(: \lambda_{0}:\right)-1+\sum_{\ell=1}^{k} \frac{\lambda_{0}}{\lambda_{\ell}} \mu_{\ell}\left(\lambda_{0}\right)
$$

One gets as well that $1-\sum_{\ell=1}^{k} \lambda_{0} / \lambda_{\ell} \mu_{\ell}\left(\lambda_{0}\right) \neq 0$ because, using the fact that the left hand side exponentials form a free set, the contrary would imply that $\lambda_{0} / \lambda_{\ell} \mu_{\ell}\left(\lambda_{0}\right)=\mu_{\ell}\left(\lambda_{0}\right)$ for all $1 \leq \ell \leq k$, which is contradictory. The right hand side of Eq. (3.15) for $\lambda=0$ is

$$
\begin{aligned}
& \left(\sum_{\ell=1}^{k} \frac{\lambda_{0}-\lambda_{\ell}}{-\lambda_{\ell}} \mu_{\ell}\left(\lambda_{0}\right)\right)^{-1} \sum_{\ell=1}^{k} \frac{\lambda_{0}-\lambda_{\ell}}{-\lambda_{\ell}} \mu_{\ell}\left(\lambda_{0}\right) \exp \left(: \lambda_{\ell}:\right) \\
= & \left(1-\sum_{\ell=1}^{k} \frac{\lambda_{0}}{\lambda_{\ell}} \mu_{\ell}\left(\lambda_{0}\right)\right)^{-1}\left(-\sum_{\ell=1}^{k} \frac{\lambda_{0}}{\lambda_{\ell}} \mu_{\ell}\left(\lambda_{0}\right) \exp \left(: \lambda_{\ell}:\right)+\sum_{\ell=1}^{k} \mu_{\ell}\left(\lambda_{0}\right) \exp \left(: \lambda_{\ell}:\right)\right) \\
= & \left(1-\sum_{\ell=1}^{k} \frac{\lambda_{0}}{\lambda_{\ell}} \mu_{\ell}\left(\lambda_{0}\right)\right)^{-1}\left(-\exp \left(: \lambda_{0}:\right)+1-\sum_{\ell=1}^{k} \frac{\lambda_{0}}{\lambda_{\ell}} \mu_{\ell}\left(\lambda_{0}\right)+\exp \left(: \lambda_{0}:\right)\right)=1 .
\end{aligned}
$$

So that the assertion is proved for $\lambda=0$ and the constants belong to the vector space spanned by our exponentials. The integration operator on exponentials, $\operatorname{Int}\left(\exp \left(: \lambda_{0}:\right)\right)=\exp \left(: \lambda_{0}:\right)-1 / \lambda_{0}$ preserves this space therefore, successive integration shows that polynomials belong to this space as well. We conclude 
that the free set of exponentials is in fact a basis of polynomials, hence of exponentials. So there exists $k=n$ complex valued functions $\mu_{\ell}(\lambda)$ such that, for all $\lambda \in \mathbb{C}$ of norm different from $2 / \delta$,

$$
\exp (: \lambda:)=\sum_{\ell=1}^{n} \mu_{\ell}(\lambda) \exp \left(: \lambda_{\ell}:\right)
$$

Therefore the previous considerations can be done for a generic $\lambda$. This shows in particular that $\mu_{\ell}(\lambda) \neq 0$ for all $\ell$ at a generic permissible point. Identifying the expansion of the constant 1 on the basis, induced by a generic $\lambda$ and by $\lambda_{0}$, one gets, for all $1 \leq m \leq n$,

$$
\begin{aligned}
\left(\sum_{\ell=1}^{n} \frac{\lambda-\lambda_{\ell}}{\lambda_{\ell}} \mu_{\ell}(\lambda)\right)^{-1} & \frac{\lambda-\lambda_{m}}{\lambda_{m}} \mu_{m}(\lambda) \\
& =\left(\sum_{\ell=1}^{n} \frac{\lambda_{0}-\lambda_{\ell}}{\lambda_{\ell}} \mu_{\ell}\left(\lambda_{0}\right)\right)^{-1} \frac{\lambda_{0}-\lambda_{m}}{\lambda_{m}} \mu_{m}\left(\lambda_{0}\right),
\end{aligned}
$$

so that $\left(\lambda-\lambda_{m}\right) \mu_{m}(\lambda)$ for $1 \leq m \leq n$ are all proportional to the same rational fraction. Solving at the origin yields the result Eq. (3.15).

It implies that the value 0 among the distinct parameters $\left(\lambda_{\ell}\right)_{0 \leq \ell \leq n}$ is permissible as well. By duality Prop 3.2, the value $\infty$ yields a well defined limit as well and in particular

$$
\varepsilon=\left(\sum_{\ell=1}^{n}\left(\lambda_{0}-\lambda_{\ell}\right) \mu_{\ell}\left(\lambda_{0}\right)\right)^{-1} \sum_{\ell=1}^{n}\left(\lambda_{0}-\lambda_{\ell}\right) \mu_{\ell}\left(\lambda_{0}\right) \exp \left(: \lambda_{\ell}:\right) .
$$

As a conclusion, any set of $n$ distinct complex parameters $\left\{\lambda_{k}\right\}_{k}$ of modulus different from $2 / \delta$ yields a basis of exponentials. By duality Eq. (3.7), the argument $\infty$ can be included as well.

REMARK 3.5. - The previous theorem can be restated using as a basis $\left(Z^{: k:} \exp (: \lambda:)\right)_{0<k<n}$ for any $\lambda \notin P_{\diamond}$, since the vector space spanned by this set is the same as the limit space spanned by $\left(\operatorname{Exp}\left(: \lambda_{k}:\right)\right)_{1 \leq k \leq n}$ when $\lambda_{k}$ all converge to $\lambda$, staying all distinct.

\section{Convexity}

4.1. Train-tracks. - We are interested only in the compact simply connected case.

Definition 4.1. - Two opposite sides in a rhombus are parallel. We will call a train-track a class of oriented edges of $\diamond$ induced by pairing the parallel opposite sides of rhombi. Let $\Theta$ this set of classes, each element $t \in \Theta$ is labelled by an angle $\theta(t) \in \mathbb{R}(\bmod 2 \pi)$ defined through any representative $e \in t$ by

TOME $132-2004-\mathrm{N}^{\mathrm{O}} 2$ 
$Z(e)=\delta \exp i \theta(t)$. The poles of the rational fraction Eq. (3.3) are contained in the set

$$
P_{\diamond}=\frac{2}{\delta} \exp -i \theta(\Theta)
$$

There is no problem to define the exponential $\exp (: \lambda:)$ for $\lambda \notin P_{\diamond}$ and the previous results extend to them. In particular, a basis of discrete exponential is given by any set of exponentials $\left\{\exp \left(: \lambda_{\ell}:\right)\right\}_{1 \leq \ell \leq n}$ of the right dimension with $\lambda_{k} \neq \lambda_{\ell}$, distinct complex (or infinite) values not in $P_{\diamond}$.

Proposition 3.2 implies that these exponentials are real on one color and pure imaginary on the other color, $\exp (: \lambda:) \in C_{\mathbb{R}}^{0}(\Gamma)+i C_{\mathbb{R}}^{0}\left(\Gamma^{*}\right)$, which can be shown by direct inspection as well.

Each color set $\Gamma$ and $\Gamma^{*}$ can be given a structure of cellular decomposition of (a subset of) the domain $U$, their edges are the diagonals of the lozenges. They are Poincaré dual to each other. The Dirichlet theorem allows to solve for a harmonic real function on the graph $\Gamma$ given some real boundary values. When the weights defining the discrete Laplacian are given by the aspect ratios of the lozenges (see Eq. (A.3) and [13]), each harmonic function on one graph can be paired with a dual harmonic function on its dual into a discrete holomorphic function, uniquely up to an additive constant. Therefore the dimension of the space of holomorphic functions is equal to half the number of points on the boundary, plus one [13]. And the number of points on the boundary is also the number of train-tracks, which makes the set of unoriented train-tracks (and another index) a likely label set for a basis of discrete holomorphic functions. The construction of this basis is the subject of Section 5 .

4.2. Convexity. - Because the rhombi are all positively oriented, a traintrack can not backtrack, does not self-intersect and two train-tracks can intersect at most once, or are opposite. A train-track $t \in \Theta$ disconnects the rest of the cellular decomposition into an initial side and a terminal side. Their role is exchanged for the opposite train-track $-t$. These sides have two parallel boundaries facing each other, say $C_{i}(t)$ and $C_{f}(t)$, such that $Z\left(C_{f}(t)\right)=\delta \exp (i \theta(\Theta))+Z\left(C_{i}(t)\right)$. A train-track $t \in \Theta$ identify pair-wise opposite edges on the positively oriented boundary, $e_{t}^{+}, e_{t}^{-} \in \partial \diamond$ with $e_{t}^{+},-e_{t}^{-} \in t$. If two train-tracks $t, t^{\prime} \in \Theta$ share the same direction, $\theta(t)=\theta\left(t^{\prime}\right)$, the edges $e_{t}^{+}, e_{t}^{-}, e_{t^{\prime}}^{+}, e_{t^{\prime}}^{-} \in \partial \diamond$, with $Z\left(e_{t}^{+}\right)=-Z\left(e_{t}^{-}\right)=Z\left(e_{t^{\prime}}^{+}\right)=-Z\left(e_{t^{\prime}}^{-}\right)$, can occur cyclically in essentially two ways: Since the train-tracks can not cross, these two pairs are not interlaced but in the order

$$
\begin{aligned}
& \left(e_{t}^{+}, \ldots, e_{t}^{-}, \ldots, e_{t^{\prime}}^{+}, \ldots, e_{t^{\prime}}^{-}, \ldots\right) \text { or } \\
& \left(e_{t}^{+}, \ldots, e_{t}^{-}, \ldots, e_{t^{\prime}}^{-}, \ldots, e_{t^{\prime}}^{+}, \ldots\right)
\end{aligned}
$$

Definition 4.2. - A map $\diamond$ is convex if the situation (4.3) only occurs. 


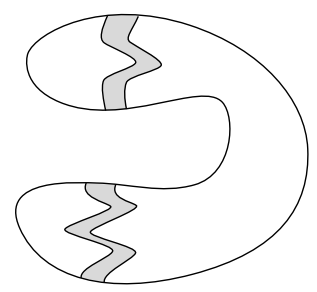

$(4.2)$

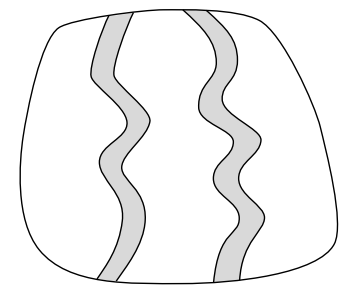

$(4.3)$

Figure 3. Non convex and convex situations.

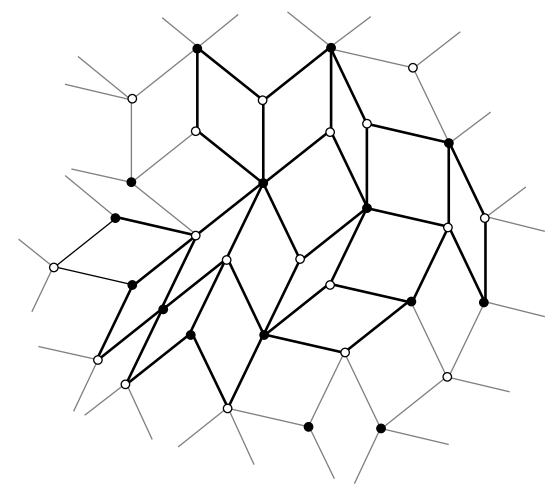

FiguRE 4. An example of a convex.

Be careful that this notion of convexity, while connected to the usual one is nevertheless different, in particular if no two train-tracks share the same slope then it is always convex, see for example Fig. 4. This notion is closely related to the spirit of $[6]$.

Proposition 4.3. - When $\diamond \subset \diamond^{\prime}$ is a portion of a larger convex compact flat critical map, convexity is equivalent to the fact that if a train-track has two rhombi in $\diamond$, then the whole portion in between is in $\diamond$ as well.

Proof. - Necessity is clear, if a train-track has two disconnected portions in the connected $\diamond$, then the latter is not convex. Conversely, if every traintrack intersects $\diamond$ in a connected line, the fact that train-tracks with equal angles don't intersect allows to continue them until the boundary of $\diamond^{\prime}$, without changing the cyclic order.

For future use, we setup the following

Definition 4.4. - Let $t \in \Theta$ a train-track, $\phi=\theta(t)$ its slope angle, $\lambda(t)=$ $(2 / \delta) \mathrm{e}^{-i \phi}$ the corresponding element in $P_{\diamond}$. Let $\diamond_{\phi}^{a}, \diamond_{\phi}^{b}, \ldots, \diamond_{\phi}^{z}$ the connected

TOME $132-2004-\mathrm{N}^{\mathrm{O}} 2$ 


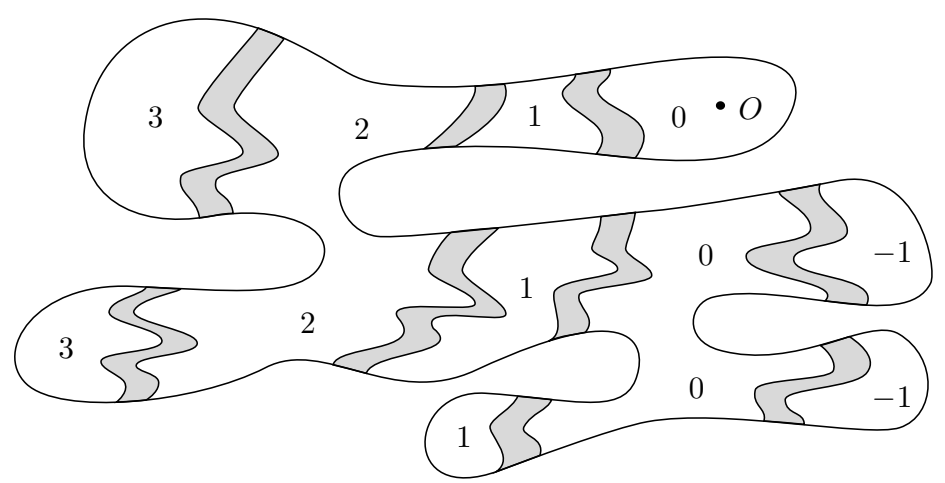

Figure 5. The level associated with an angle.

components of $\diamond$ minus the rhombi in $\theta^{-1}(\phi)$, having the same slope as $t$. These components are ordered into a chain of initial and terminal sides, forming an oriented tree ${ }^{(1)}$. Each component has a given level $d_{\phi}$ counting the algebraic number of times these train-tracks were crossed from the origin. To each train-track $t$, we associate the connected component on its terminal side and denote it $\diamond^{t}$. We define likewise $d_{\phi}(t)$.

REMARK 4.5. - The rational fraction $\exp (: \mu: x)$, for $x \in \diamond^{t}$ has a pole of order $d_{\phi}(t)$ at $\mu=\lambda(t)$ and a zero of same order at $\mu=-\lambda(t)$ if $d_{\phi}(t)>0$, or a zero of order $-d_{\phi}$ at $\mu=\lambda(t)$ and a pole of same order at $\mu=-\lambda(t)$ if $d_{\phi}(t)<0$.

The level associated with an angle $\phi$ is opposite to the level associated with $-\phi, d_{-\phi}=-d_{\phi}$ : A zero at $\mu=\lambda_{0}$ for $\exp (: \mu: x)$ is equivalent to a pole of the same order at $\mu=-\lambda_{0}$. The two connected components which are separated by a given train-track $t$ are $\diamond^{t}$ and $\diamond^{-t}$. See Fig. 5 for an example.

\subsection{Proof of the main result}

Proof of Theorem 1. - Consider a point $x \in \diamond_{0}$ and a path $\gamma$ form the origin to $x$. This path can be so chosen that the train-tracks it crosses are crossed only once. Its length is the combinatorial distance $d(x, O)$ between $x$ and the origin. The point $x$ is uniquely determined by the set $\Theta_{x}$ of train-tracks the path crosses. Indeed,

$$
x=\sum_{t \in \Theta_{x}} \delta \mathrm{e}^{i \theta(t)}
$$

(1) Which is not a total ordering if $\diamond$ is not convex. 
where we have written $x$ in place of its $Z$-image $Z(x)$. Given a basis $\left\{\exp \left(: \lambda_{\ell}:\right)\right\}_{1 \leq \ell \leq n}$ of discrete exponentials such as in Prop. 2, the rational fraction Eq. (3.3) in $\lambda$, equals the basis expansion Eq. (3.15),

$$
\begin{aligned}
\exp (: \lambda: x) & =\prod_{t \in \Theta_{x}} \frac{1+\frac{1}{2} \lambda \delta \mathrm{e}^{i \theta(t)}}{1-\frac{1}{2} \lambda \delta \mathrm{e}^{i \theta(t)}} \\
& =\left(\sum_{\ell=1}^{n} \frac{\lambda_{0}-\lambda_{\ell}}{\lambda-\lambda_{\ell}} \mu_{\ell}\left(\lambda_{0}\right)\right)^{-1} \sum_{\ell=1}^{n} \frac{\lambda_{0}-\lambda_{\ell}}{\lambda-\lambda_{\ell}} \mu_{\ell}\left(\lambda_{0}\right) \exp \left(: \lambda_{\ell}: x\right) .
\end{aligned}
$$

The dependency in $x$ of the basis expansion is solely through the complex numbers $\exp \left(: \lambda_{\ell}: x\right)$. It is immediate to see that the poles in front of them are canceled by zeros of the prefactor so that

$$
\exp (: \lambda: x)=\frac{P_{x}(\lambda)}{Q(\lambda)}
$$

with $P_{x}$ and $Q$ are polynomials, both of degree $n-1$, the former depending on $x$ but not the latter. Some zeros of $P_{x}$ cancel some of the zeros of $Q$, leaving $d(x, O)$ poles, counted with multiplicities. Each pole is of the form $\lambda=(2 / \delta) \mathrm{e}^{-i \theta(t)}$ and corresponds to one of the train-tracks $t$ between $x$ and the origin.

If the train-tracks angles are all different, considering all the paths starting at the origin, we see that each train-track such that the origin is on its initial side contributes to a zero of $Q$. Therefore its degree is at least the number of such train-tracks. This number is equal to the unoriented train-tracks, that is to say half the number of vertices on the boundary. Therefore

$$
n \geq \frac{|\partial \diamond|}{2}+1
$$

The right hand side is the dimension of the space of discrete holomorphic functions, which is an upper bound for $n$, yielding the result in that case.

If some train-tracks share the same angle, they don't contribute to different zeros of $Q$ but possibly to a higher order for the same zero.

It is a matter of definition to check that when a point $x$ is in a connected component $\nabla_{\phi}^{\ell}$ at a level $d_{\phi}>0$, the train-tracks of angle $\phi$ encountered from the origin, contribute to a zero at $\lambda=(2 / \delta) \mathrm{e}^{-i \phi}$ of order $d_{\phi}$ in $Q(\lambda)$. When $d_{\phi}<0$, the zero is at $\lambda=-(2 / \delta) \mathrm{e}^{-i \phi}$.

Convexity is equivalent to the fact that there is, for each possible slope, only one connected component at a given level. Therefore, in that case as well, each (unoriented) train-track contributes to exactly one zero of the numerator $Q$, counting multiplicities. The same counting yields the result.

TOME $132-2004-\mathrm{N}^{\mathrm{O}} 2$ 


\section{Special Exponentials}

We are going to define the special exponentials $\exp _{t}\left(: \lambda_{0}: x\right)$ for $\lambda_{0} \in P_{\diamond}$, one for every train-track $t$ of slope $2 / \lambda_{0}=\delta \mathrm{e}^{i \theta(t)}$, that is to say one for every possible pole (and multiplicity) in $\lambda$ of the exponential $\exp (: \lambda: x)$ at a given vertex $x \in \nabla_{0}$. Richard Kenyon's contour integral 1.2 comes from a sum of residues over the poles. In the same fashion, a contour integral of any holomorphic measure in the $\lambda$ space can be concentrated as a weighted sum over these special exponentials.

5.1. Definition. - Let $t \in \Theta$ a train-track, $\phi=\theta(t)$ its slope angle, $\lambda_{0}=$ $\lambda(t) \in P_{\diamond}$, and $\diamond_{\phi}^{a}, \diamond_{\phi}^{b}, \ldots, \diamond_{\phi}^{z}$ the associated connected components of $\diamond$.

Consider the rational fraction $\exp (: \lambda: x)$, at a point $x \in \diamond_{\phi}^{\ell}$ on a connected component at level $d_{\phi}(\ell)>0$. Choose an origin $O_{\phi}^{\ell} \in \diamond_{\phi}^{\ell}$ for each connected component $\ell$. It defines $\exp _{\ell}(: \lambda: x):=\exp \left(: \lambda:\left(x-O_{\phi}^{\ell}\right)\right)_{\diamond_{\phi}^{\ell}}$, free of zeros and poles at $\lambda= \pm \lambda_{0}$ for every point $x \in \diamond_{\phi}^{\ell}$. We extend it by zero to other connected components. The exponential $\exp \left(: \lambda:\left(x-O_{\phi}^{\ell}\right)\right)$ has a well defined limit on the components of level lower than $d_{\phi}(\ell)$, it is the null function because on each component it contains a factor of the form $\left(\lambda-\lambda_{0}\right)$, therefore the Cauchy-Riemann equation is fulfilled across the train-track to the previous components. On the next components however, $\exp \left(: \lambda:\left(x-O_{\phi}^{\ell}\right)\right)$ diverges since it contains the factor $\left(\lambda-\lambda_{0}\right)^{-1}$. Continuing it by zero yields $\exp _{\ell}(: \lambda: x)$ which is no longer a discrete holomorphic function because it fails to fulfill the Cauchy-Riemann equation across the train-track to the next components.

Proposition 5.1. - For every connected component $\diamond_{\phi}^{\ell}$, there exists a unique set of complex numbers $\left(\mu_{m}\right)$, one for each connected component $\diamond_{\phi}^{m}$ such that the following piece-wise discrete holomorphic function

$$
\sum_{m} \mu_{m} \frac{\mathrm{d}^{d_{\phi}(m)-d_{\phi}(\ell)}}{\mathrm{d} \lambda^{d_{\phi}(m)-d_{\phi}(\ell)}} \exp _{m}(: \lambda: x)
$$

is a well defined discrete holomorphic function for $\lambda=\lambda_{0}$, on the whole map $\diamond$, with

1) $\mu_{\ell}=1$ and

2) $\mu_{m}=0$ for every component which can not be reached from $\diamond_{\phi}^{\ell}$ by a path staying above level $d_{\phi}(\ell)$.

DEFINITION 5.2. - We call the previous discrete holomorphic function a special exponential and denote it $\exp _{t}\left(: \lambda_{0}: x\right)$ for the train-track $t$ such that $\diamond_{\phi}^{\ell}=\diamond^{t}$. 


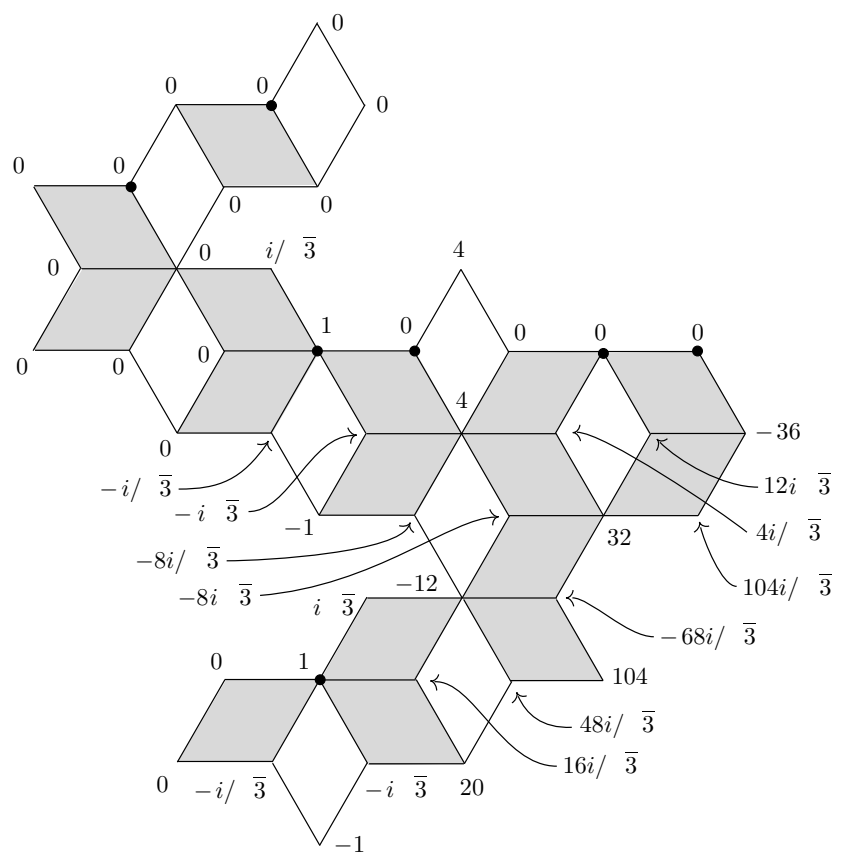

FIGURE 6. A special exponential $\exp (: 1:)$ on the standard triangular/hexagonal lattice with $\delta=2$. The base point for each component is marked by a solid dot and the value at these points is 0 .

Equation (3.6) defines $Z^{: k} \exp (: \lambda: Z)$ as the successive derivatives of the discrete exponential with respect to $\lambda$. It would be confusing to note the special exponential $\sum_{m} \mu_{m} x^{: d_{\phi}(m)-d_{\phi}(\ell):} \exp _{m}(: \lambda: x)$.

EXAMPLE 5.3. - The special exponential corresponding to the central zone at level 1 in Fig. 5 will be null on the components at levels 0 and -1 but also on the components at level 1 corresponding to the lower leg of the figure. It is equal to the special exponential corresponding to the other component at level 1 on the upper leg, up to a global multiplicative factor (and provided the origins in the various components are kept the same).

The lattices have few different slopes so that the connected components appearing above are reduced to thin areas, see Fig. 6 for an explicit example on the triangular/hexagonal lattice. The extreme case is the standard square lattice where they are reduced to lines.

The demonstration uses the following 
Lemma 5.4. - For every connected component $\diamond_{\phi}^{\ell}$, and degree $d<d_{\phi}(\ell)$, there exists a unique set of complex numbers $\left(\nu_{k}\right)_{0 \leq k<d_{\phi}(\ell)-d}$ such that

$$
\left(\lambda-\lambda_{0}\right)^{d} \exp (: \lambda: x)-\sum_{k=0}^{d_{\phi}(\ell)-d-1} \nu_{k}\left(\lambda-\lambda_{0}\right)^{d-d_{\phi}(\ell)+k} \frac{\mathrm{d}^{k}}{\mathrm{~d} \lambda^{k}} \exp _{\ell}(: \lambda: x)
$$

has no poles and no zero at $\lambda=(2 / \delta) \mathrm{e}^{-i \phi}$ on $\diamond_{\phi}^{\ell}$. Moreover, it is proportional to $\mathrm{d}^{d_{\phi}(\ell)-d} \exp _{\ell}(: \lambda: x) / \mathrm{d} \lambda^{d_{\phi}(\ell)-d}$.

Proof. - Given $x \in \diamond_{\phi}^{\ell}$, the rational fraction $\left(\lambda-\lambda_{0}\right)^{d} \exp (: \lambda: x)$ has a pole at $\lambda=(2 / \delta) \mathrm{e}^{-i \phi}$ of order $d_{\phi}(\ell)-d$ while $\exp _{\ell}(: \lambda: x)$ is free of zero or pole there. A Taylor expansion at $\lambda=\lambda_{0}$ of the following product gives

$$
\begin{gathered}
\left(\lambda-\lambda_{0}\right)^{d_{\phi}(\ell)} \exp (: \lambda: x)=\left(\lambda-\lambda_{0}\right)^{d_{\phi}(\ell)} \exp \left(: \lambda: O_{\phi}^{\ell}\right) \exp _{\ell}(: \lambda: x) \\
=\sum_{k=0}^{d_{\phi}(\ell)-d}\left(\lambda-\lambda_{0}\right)^{k} F_{k}(\lambda) \frac{\mathrm{d}^{k}}{\mathrm{~d} \lambda^{k}} \exp _{\ell}(: \lambda: x)+o\left(\lambda-\lambda_{0}\right)^{d_{\phi}(\ell)-d}
\end{gathered}
$$

where $F_{k}(\lambda)$ is a rational fraction with no pole and no zero at $\lambda=\lambda_{0}$. Defining $\nu_{k}:=F_{k}\left(\lambda_{0}\right)$, which does not depend on $x$, one gets the result.

Proof of Proposition 5.1. - We use the lemma on every connected component with $d=d_{\phi}(\ell)$. Writing as previously the exponential in sums of different rational fractions with poles at $\lambda=\lambda_{0}$ of various orders, shows that each order independently belongs to the space of usual exponentials and has to fulfill the Cauchy-Riemann equation when $\lambda$ tends to $\lambda_{0}$. It is a matter of course inside each connected component, but it is also the case across the gaps made by the train-tracks between these components. In particular the degree 0 provides a well-defined discrete holomorphic function, which satisfies the condition 1), $\mu_{\ell}=1$. Moreover the exponential $\exp \left(: \lambda:\left(x-O_{\phi}^{\ell}\right)\right)$ for $x \in \diamond^{m}$ with $d_{\phi}(m)<d_{\phi}(\ell)$, since it contains the factor $\left(\lambda-\lambda_{0}\right)$, has zero as a limit when $\lambda$ tends to $\lambda_{0}$ for $x \in \diamond^{m}$ with $d_{\phi}(m)<d_{\phi}(\ell)$. Therefore we can discard all the components at level less than $d_{\phi}(\ell)$ (which correspond to integration with respect to $\lambda$ ). Then every connected subtree of the partial ordering of the connected components staying above level $d_{\phi}(\ell)$ can be continued by zero on the rest of the map. Which proves the fact that the second requirement can be met.

A by-product of the proof is the following

Corollary 5.5. - For each attained level $d \in d_{\phi}(\Theta)$, there exists a linear combination

$$
\sum_{t / d_{\phi}(t)=d} \pi_{t} \exp _{t}(: \lambda: x)
$$

BULletin DE LA SOCiÉtÉ MATHÉmATiQUE DE FRANCE 
unique up to a multiplicative constant, which belongs to the space of usual discrete exponentials.

5.2. Basis. - We have now to show that they form a generating set for holomorphic functions. As we pointed out in the Example 5.3, the special exponentials associated with different train-tracks may coincide. In order to get the right dimension, we have to select half of them. We defined $\diamond^{t}$ as the connected component on the terminal side of $t$.

DeFInITION 5.6. - A train-track $t$ is positively oriented if a path of shortest length from the origin to a point $x \in \diamond^{t}$ contains an edge in $t$.

It is equivalent to the fact that a path of shortest length from the origin to a point $x \in \diamond^{-t}$, the previous component in the direction of $t$, contains no edge in $t$.

The great interest of such a notion is that when a train-track $t$ is positively oriented, the associated special exponential $\exp _{t}(: \lambda: x)$ is null on the subtree on the initial side of $t$, which contains the origin. This allows for the construction of a very natural basis starting from the origin.

THEOREM 3. - The set of all special exponentials for positively oriented traintracks and the constant function 1 form a basis of discrete holomorphic functions.

This is done simply by expressing the generic exponential on this basis, reconstructing its needed poles out of the special exponentials:

LEMMA 5.7. - Let $\Theta_{O}^{+}$the set of positively oriented train-tracks from the origin $O$. There exists a unique set of complex numbers $\left(\kappa_{t}\right)_{t \in \Theta_{O}^{+}}$such that

$$
\exp (: \mu:)=1+\sum_{t \in \Theta_{O}^{+}} \kappa_{t}(\mu-\lambda(t))^{-d_{\theta(t)}} \exp _{t}(: \lambda(t):) .
$$

Proof. - The two sides of Eq. (5.5) are both discrete holomorphic functions for generic $\mu$, and at each point are rational fractions of $\mu$ with the same set of poles, $\left\{\lambda\left(t_{k}\right)\right\}$ associated with train-tracks $t_{k}$ between the origin and $x$, and orders $d_{\theta\left(t_{k}\right)}$, uniquely defining $x=\sum_{t_{k}} \delta e^{i \theta\left(t_{k}\right)}$. Their value at the origin are both equal to 1 .

Consider $\Theta_{O}^{k}$ the set of positively oriented train-tracks which can be reached in $k$ steps from the origin. Let $\diamond^{k}$ the sub-complex of $\diamond$ spanned by continuous paths from the origin whose edges are in $\Theta_{O}^{k}$ (we will say spanned by $\Theta_{O}^{k}$ ). Its vertices are a sup-set of the set of vertices at a combinatorial distance less than $k+1$ from the origin.

Suppose that we have proven Eq. (5.5) for the sub-complex $\diamond^{d}$, assigning a value $\kappa_{t}$ to every $t \in \diamond^{d}$. All the other positively oriented train-tracks are such

TOME $132-2004-\mathrm{N}^{\mathrm{O}} 2$ 
that their special exponential is null on $\diamond^{d}$ therefore it makes sense to restrict Eq. (5.5) to $\diamond^{d}$. The equality obviously holds for $d=0$ where $\diamond^{0}=\{O\}$.

A point $x \in \diamond^{d+1} \backslash \diamond^{d}$ is characterized by a new train-track $t \in \Theta_{O}^{d+1} \backslash \Theta_{O}^{d}$. The equation Eq. (5.5) provides a linear determination for $\kappa_{t}$ :

$$
\begin{aligned}
\kappa_{t}:=\lim _{\mu \rightarrow \lambda(t)}(\mu-\lambda(t))^{d_{\theta(t)}}\{ & \exp (: \mu:)-1 \\
& \left.\quad-\sum_{u \in \Theta_{O}^{k}} \kappa_{u}(\mu-\lambda(u))^{-d_{\theta(u)}} \exp _{u}(: \lambda(u):)\right\} .
\end{aligned}
$$

Since $\exp _{t}(: \lambda(t):)$ is null on $\diamond^{d}$, Eq. (5.5) restricted to $\diamond^{d}$ is not perturbed by this assignation. The right hand side of Eq. (5.5) is discrete holomorphic on the set spanned by $\Theta_{O}^{d} \cup\{t\}$. On the other hand, the Cauchy-Riemann equation allows to solve uniquely for the value at every other vertex spanned by $\Theta_{O}^{d} \cup\{t\}$, therefore Eq. (5.5) holds for all these vertices, showing that the choice of the vertex $x$ is irrelevant. Another vertex $y \in \diamond^{d+1} \backslash \diamond^{d}$ defining another traintrack $t^{\prime}$ defines $\kappa_{t^{\prime}}$ in a similar way. If $t \cap t^{\prime} \in \diamond^{d+1}$, one checks easily that the order with which $\kappa_{t}$ and $\kappa_{t^{\prime}}$ are chosen is irrelevant. It is even more so if their intersection is empty or if this intersection does not belong to $\diamond^{d+1}$.

Proceeding for every train-track in $\diamond^{d+1} \backslash \diamond^{d}$, we assign uniquely a value $\kappa_{u}$ to every train-track $u \in \Theta_{O}^{d+1} \backslash \Theta_{O}^{d}$ and prove Eq. (5.5) for the sub-complex $\diamond^{d+1}$. By induction we prove it for the whole map $\diamond$.

Proof of Theorem 3. - Uniqueness in the lemma shows that the set of special exponentials is a free set. The number of positively oriented train-tracks plus one (for the constant function 1) is equal to the dimension of discrete holomorphic functions.

\section{A. Appendix: additional properties}

A.1. Eigenvalues of integration. - The polynomials on $\diamond$ are finite dimensional, hence there exists a minimal degree for which $Z^{: n}$ i is linked with the previous monomials.

Proposition A.1. - Let $P_{Z}=\sum_{k=1}^{n} a_{k} Z^{: k}=0$ the minimal polynomial of the map $Z$. The eigenvalues of the integration operator are the roots of the polynomial $Q=\sum_{k=1}^{n} k ! a_{k} \lambda^{k}$,

$$
\operatorname{Spec}(\operatorname{Int})=Q^{-1}(0)
$$

Proof. — In the basis $\left(Z^{: k:} / k !\right)_{0 \leq k<n}$, where $\operatorname{Int}\left(Z^{: k:} / k !\right)=Z^{: k+1:} /(k+1)$ !, up to $Z^{: n:} / n !=-\sum_{k=1}^{n-1} k !\left(a_{k} / n !\right) a_{n} Z^{: k} / k$ !, the integration operator has the 
following matrix representation

$$
\operatorname{Int}=\left(\begin{array}{cccccc}
0 & \ldots & \ldots & \ldots & 0 & 0 \\
1 & \ddots & & \vdots & -a_{1} / n ! a_{n} \\
0 & \ddots & \ddots & \vdots & -2 ! a_{2} / n ! a_{n} \\
\vdots & \ddots & \ddots & \ddots & \vdots & \vdots \\
\vdots & & \ddots & \ddots & 0 & -(n-2) ! a_{n-2} / n ! a_{n} \\
0 & \ldots & \ldots & 0 & 1 & -(n-1) ! a_{n-1} / n ! a_{n}
\end{array}\right)
$$

and its characteristic polynomial is $Q$. The minimal polynomial $P_{Z}$ can be normalized so that $P_{Z}^{\prime}(Z)=\varepsilon$, that is to say $a_{1}=1$. The eigenvector associated with 0 is $\varepsilon$.

A.2. Derivation. - Any linear normalization of the degree of freedom $\lambda$ in Eq. (2.6) defines a derivation operator $\mathrm{d} / \mathrm{d} Z$. For example, if $\left(O, y_{k}, x_{k}, y_{k+1}\right)$ in $\nabla_{2}$ are the quadrilaterals adjacent to the origin, with the weights of the discrete Laplacian $\rho\left(O, x_{k}\right)=-i\left(Z\left(y_{k+1}\right)-Z\left(y_{k}\right)\right) /\left(Z\left(x_{k}\right)-Z(O)\right)>0$ controlling their aspect ratio, then the normalization

$$
\sum_{k} \rho\left(O, x_{k}\right)\left(\frac{f\left(y_{k+1}\right)-f\left(y_{k}\right)}{Z\left(y_{k+1}\right)-Z\left(y_{k}\right)}-\lambda\right)=0,
$$

states that the derivative at the origin is the mean value of the nearby face derivatives.

Because the derivation operator can have only a finite number of different eigenvalues, the derivatives of exponentials are not always exponentials, so that

$$
\exp (: \lambda: Z)^{\prime}=\lambda \exp (: \lambda: Z)-f_{Z}(\lambda) \varepsilon
$$

with a non trivial function $f_{Z}$, depending on the normalization.

Proposition A.2. - Let $\left(\lambda_{1}, \ldots, \lambda_{n}\right) \in \mathbb{C}^{n}$ distinct complex values of norm different from $2 / \delta$, which define a basis $\left\{\exp \left(: \lambda_{k}:\right)\right\}$ of exponentials on $\diamond$ and a certain normalization of the derivation operator, that is to say $n$ fixed values $f_{Z}\left(\lambda_{\ell}\right) \in \mathbb{C}$. Then, the normalization function is the following rational fraction:

$$
f_{Z}(\lambda)=\sum_{\ell=1}^{n} \mu_{\ell}(\lambda)\left(\lambda-\lambda_{\ell}+f_{Z}\left(\lambda_{\ell}\right)\right)
$$

in terms of the coordinates of $\exp (: \lambda:)=\sum_{\ell=1}^{n} \mu_{\ell}(\lambda) \exp \left(: \lambda_{\ell}:\right)$ on the basis.

Each coordinate $\mu_{\ell}(\lambda)$ is a rational fraction given in Eq. (3.15). By Eq. (A.4), the zeros of the rational fraction $f_{Z}$, if their norm is different from $2 / \delta$, give all the eigenvectors of the derivation operator. Uniqueness of the exponential implies that these eigenvalues are always non degenerate.

TOME $132-2004-\mathrm{N}^{\mathrm{O}} 2$ 
In [12], we defined a normalization adapted to polynomials of finite degree. It is the least interesting in that respect since its only eigenvalue is 0 , the unique eigenvector associated being the constant 1 . We define a normalization adapted to a basis of exponentials:

Definition A.3. - Let $\left(\lambda_{1}, \ldots, \lambda_{n}\right) \in \mathbb{C}^{n}$ distinct complex values of norm different from $2 / \delta$, which define a basis $\left\{\exp \left(: \lambda_{k}:\right)\right\}$ of exponentials on $U$. We normalize the derivation operator Eq. (2.6) such that it is diagonal in this basis.

Because of Eq. (3.16), the normalization adapted to polynomials is associated with a sequence $\left(\lambda_{\ell}^{k}\right)_{k \in \mathbb{N}}$ of $n$ parameters $\lambda_{1}^{k} \gg \lambda_{2}^{k} \gg \cdots \gg \lambda_{n}^{k}$ converging to zero at different rates with $k$, for example $\lambda_{\ell}^{k}=k^{-\ell}$.

A.3. Refinement. - In a refining sequence of critical maps, where the size $\delta$ goes to zero, the length of the shortest discrete path between two fixed points $O, x$ on the surface remains of order of their euclidian distance $|x|$. Therefore, expanding $\exp (\log (\exp (: \lambda: x)))$ we get

$$
\exp (: \lambda: x) \underset{\delta \rightarrow 0}{=} \exp (\lambda x)+O\left(\lambda^{3}|x|^{3} \delta^{2}\right) .
$$

A.4. Change of base point. - The change of base point for a polynomial is not as simple as the Pascal binomial formula of the continuous case [12]. Nevertheless, for exponentials, if $\zeta=a(Z-b)$ :

$$
\sum_{k=0}^{\infty} \frac{\lambda^{k}}{k !} \zeta^{: k:}(x)=\exp _{Z}(: \lambda: b) \sum_{k=0}^{\infty} \frac{(a \lambda)^{k}}{k !} Z^{: k:}(x) .
$$

A.5. Immersion. - By inspecting the map

$$
\mathbb{C} \longrightarrow \mathbb{C}, \quad z \longmapsto \frac{1+z}{1-z}
$$

one sees that the quadrilateral

$$
\left(1, \frac{1+z^{\prime}}{1-z^{\prime}}, \frac{1+z}{1-z} \cdot \frac{1+z^{\prime}}{1-z^{\prime}}, \frac{1+z}{1-z}\right)
$$

with $|z|=\left|z^{\prime}\right|<1$ is mapped to a positively oriented quadrilateral whose diagonals cross at a right angle. It shows that the exponential $\exp (: \lambda:)$ with $|\lambda|<2 / \delta$ maps each rhombus to a convex quadrilateral. Therefore it is a locally injective map.

\section{BIBLIOGRAPHY}

[1] Agafonov (S.I.) \& Bobenko (A.I.) - Discrete $Z^{\gamma}$ and Painlevé equations, Internat. Math. Res. Notices (2000), no. 4, pp. 165-193. 
[2] Bobenko (A.I.) - Discrete conformal maps and surfaces, in Symmetries and integrability of difference equations (Canterbury, 1996), Cambridge Univ. Press, Cambridge, 1999, pp. 97-108.

[3] Bobenko (A.I.) \& Pinkall (U.) - Discrete isothermic surfaces, J. reine angew. Math., t. 475 (1996), pp. 187-208.

[4] Bobenko (A.I.) \& SeILer (R.), eds. - Discrete integrable geometry and physics, Oxford Lecture Series in Mathematics and its Applications, vol. 16, The Clarendon Press Oxford University Press, New York, 1999.

[5] Bobenko (A.I.) \& SURIS (YU.B.) - Integrable systems on quad-graphs, http://arXiv.org/abs/nlin.SI/0110004, 2001.

[6] Colin de Verdière (Y.), Gitler (I.) \& Vertigan (D.) - Réseaux électriques planaires. II, Comm. Math. Helv., t. 71 (1996), no. 1, pp. 144167.

[7] Duffin (R.J.) - Basic properties of discrete analytic functions, Duke Math. J., t. 23 (1956), pp. 335-363.

[8] _ Potential theory on a rhombic lattice, J. Comb. Theory, t. 5 (1968), pp. 258-272.

[9] Hertrich-Jeromin (U.) - Introduction to Möbius Differential Geometry, London Mathematical Society Lecture Note Series, vol. 300, Cambridge University Press, Cambridge, 2003.

[10] Kenyon (R.) - The Laplacian and Dirac operators on critical planar graphs, Invent. Math., t. 150 (2002), no. 2, pp. 409-439, http://arXiv. org/abs/math-ph/0202018.

[11] Mercat (CH.) - Discrete Period Matrices and Related Topics, http:// arXiv.org/abs/math-ph/0111043.

[12] Discrete Polynomials and Discrete Holomorphic Approximation, http://arXiv.org/abs/math-ph/0206041.

[13] _ Discrete Riemann surfaces and the Ising model, Comm. Math. Phys., t. 218 (2001), no. 1, pp. 177-216. 